\title{
Barriers to and incentives for achieving partograph use in obstetric practice in low- and middle-income countries: a systematic review
}

\author{
Elizabeth Ollerhead ${ }^{*}$ and David Osrin
}

\begin{abstract}
Background: The partograph is a graphic display of the progress of labour, recommended by the World Health Organization, but often underused in practice in low- and middle-income countries. We were interested in going beyond demonstration of potential efficacy - on which the existing literature concentrates - through a systematic review to identify barriers to and incentives for achieving partograph use.

Methods: We searched Ovid MEDLINE, Ovid Maternity and Infant Care, POPLINE, Web of Science, and Scopus, from $1^{\text {st }}$ January 1994 to $30^{\text {th }}$ September 2013, using the term 'partogra*' to include 'partograph', 'partogram', or 'partogramme'. The selection criteria were for primary or secondary research describing barriers to and incentives for partograph use in low- and middle-income countries, in English, reported in peer-reviewed publications since 1994. Thematic analysis of text on partograph use was applied to a commonly used framework for change in clinical practice, with levels describing the innovation, the individual professional, the woman, and social, organisational, economic and political contexts.
\end{abstract}

Results: Reported barriers to and incentives for partograph use related to the partograph itself, professional skills and practice, clinical leadership and quality assurance, and the organisational environment within the wider provision of obstetric care. Neither the evidence base for its effectiveness, nor its credibility, was reported as a barrier to use.

Conclusion: Identifying and addressing local barriers and incentives in low- and middle-income countries, based on those in published research, could inform strategies to improve partograph use. Emerging technologies could be used to address some barriers. The thresholds for essential maternity care at which the partograph adds value should be further evaluated.

Keywords: Obstetrics, Labor, Obstetric, Health knowledge, Attitudes, Practice, Quality of health care, Developing countries, Review, Systematic

\section{Background}

A graphic display of the progress of labour, the partograph helps skilled birth attendants to recognise emerging difficulties and take action according to a clinical management protocol. Partography can reduce the burden of obstructed labour as a cause of maternal and fetal death, obstetric fistula, and fetal hypoxia, birth trauma and infection [1]. Requiring only a printed sheet to chart what should be routine care, the partograph would seem to be an appropriate technology with a clear place in maternity care

*Correspondence: elizabeth.ollerhead.09@alumni.ucl.ac.uk

UCL Institute for Global Health, 30 Guilford Street, London WC1N 1EH, UK in low- and middle-income countries (LMICs). It is variably used, however, and often reported as substantially underused [2].

One response to this shortfall is to demonstrate potential efficacy and effectiveness. The WHO prospective trial in South East Asia suggested that partography with a management protocol reduced prolonged labour and promoted more appropriate obstetric assessment and intervention [3], although a Cochrane review of trials in variably resourced settings found insufficient evidence to either support or discourage its use [4]. Some gaps remain in the evidence base, and the assumption of universal 
relevance in a diversity of contexts and for a diversity of women is still debated [5]. Our concern is less that the partograph might have failed tests of efficacy, and more that it has been lost in translation. Wide variation in use between countries has been reported, even in comparisons at similar levels of health systems [2], suggesting that contextual factors may be important influences. "One of the most consistent findings in health services research is the gap between best practice (as determined by scientific evidence), on the one hand, and actual clinical care, on the other" [6]. Because we wanted to understand the literature on usage as a first step to addressing practice [7], we did a systematic review of barriers to and incentives for partography in LMICs. If we assume that the partograph is a technology with an evidence-based role in obstetric care, its adoption, regular use, and quality fall into the realm of change in practice. Healthcare workers are well aware of the complexities inherent in changing practices, and implementation theory is increasingly contributing to managing change [8,9]. In framing the findings of our review, we used a classification suggested by Grol and Wensing [6] that has pedigree in analyses of healthcare by, among others, the UK National Institute for Health and Clinical Excellence [10] and the Australian National Institute of Clinical Studies [11].

\section{Methods}

The study was developed as a dissertation based on an iterative process rather than a protocol. Journal publications about partography, in English, were identified from 1994, when the composite WHO partograph was recommended. We selected 1994 as the starting point because it was a clear policy moment following the updated WHO guidance. After partograph development reported by Philpott [12], early studies researched efficacy, application to different populations, and clinical scenarios and outcomes. Most publications related to high-income countries, and descriptions of issues around use in practice in LMICs became a more common theme around 2000.

We searched Ovid MEDLINE, Ovid Maternity and Infant Care, POPLINE, Web of Science, and Scopus, from 1st January 1994 to 30th September 2013, using the term 'partogra"' to include the words 'partograph(s)', 'partogram(s)' or 'partogramme(s)' (1 partogra".mp. [mp = title, abstract, original title, name of substance word, subject heading word, keyword heading word, protocol supplementary concept, rare disease supplementary concept, unique identifier. 2 limit 1 to (english language and $y r=" 1994$-Current")]). Search results were imported into EndNote X5. Primary or secondary research was selected, referring to LMICs using the 2012 World Bank
Classification. Publications that were not research or reviews - including reports, books, news articles, editorials, and letters - were excluded due to limited detail on partograph use. We chose not to include theses and conference papers. Publications reporting reasons for partograph use and non-use were included in the synthesis, while those that only quantified use were excluded. We copied text about barriers and incentives to partograph use into a table and rearranged it into provisional themes, which identified that reported issues for use were predominantly about individual professionals in the context of their working environment, rather than concerns about the evidence-base for partographs. The provisional themes were reconciled with the practice change framework [6]. Table 1 outlines the thematic classification of barriers to and incentives for partograph use, based on the framework by Grol and Wensing [6]. The identification, selection and analysis were carried out by one reviewer (EO). Although bias presumably exists in the sense that publications were more likely to arise from situations in which the introduction of partography had been unsuccessful, the qualitative nature of the data meant that we were unable to quantify risk.

\section{Role of the funding source}

The sponsor had no role in the study design, data collection, analysis, interpretation or writing of the article. EO had access to all study data and responsibility for the decision to submit for publication.

\section{Results}

There were 346 references after removal of duplicates. All 346 abstracts were screened to select publications in which partograph use was described in practice. $199 \mathrm{pa}-$ pers were excluded at screening - 95 only related to high-income countries, 63 were not primary or secondary research in peer-reviewed publications, and 41 only mentioned the partograph as a measure for testing other clinical interventions. 147 publications were selected for full review. 73 were excluded because they provided no information on barriers or incentives for use. The sequence of review is summarised in the PRISMA [13] Flow Diagram in Figure 1.

There were 74 publications for narrative synthesis on partograph use: 64 were studies of healthcare, including 18 audits, 18 surveys, 20 practice assessments, and 8 training evaluations; 10 were reviews. Of 59 primary studies from a single country, 43 were from Sub-Saharan Africa, 9 from South Asia, and the rest from other Asian countries.

\section{Innovation}

The innovation may be a barrier or an incentive, depending on its perceived advantages in terms of practice, 
Table 1 Classification of barriers to and incentives for partograph use in low and middle income countries

\begin{tabular}{|c|c|c|c|}
\hline Theme [6] & Characteristics [6] & $\begin{array}{l}\text { Incentives identified in thematic } \\
\text { analysis }\end{array}$ & Barriers identified in thematic analysis \\
\hline \multirow[t]{5}{*}{ The innovation itself } & Advantages in practice & Monitoring labour & Availability \\
\hline & Feasibility & Continuity of care & Graphing skills \\
\hline & Credibility & & Language \\
\hline & Accessibility & & Literacy \\
\hline & Attractiveness & & Many partograph versions \\
\hline \multirow[t]{6}{*}{ Individual professional } & Awareness & Skilled birth attendants & Awareness \\
\hline & Knowledge & Positive attitude & Knowledge \\
\hline & Attitude & & Skills \\
\hline & Motivation to change & & Commitment \\
\hline & Behavioural routines & & Confidence \\
\hline & & & Negative attitude \\
\hline \multirow[t]{4}{*}{ Patient (Woman) } & Knowledge & Knowledge & Late admission \\
\hline & Skills & & \\
\hline & Attitude & & \\
\hline & Compliance & & \\
\hline \multirow[t]{9}{*}{ Social context } & Opinion of colleagues & Leadership & Inter-professional barriers \\
\hline & Culture of the network & Staff involvement & Lack of: \\
\hline & Collaboration & Supervision & - Engagement \\
\hline & Leadership & Monitoring & - Leadership \\
\hline & & Audit & - Role models \\
\hline & & Evaluation & - Facilitation \\
\hline & & & - Monitoring \\
\hline & & & - Evidence-based practice \\
\hline & & & Retrospective documentation \\
\hline \multirow[t]{8}{*}{ Organisational context } & $\begin{array}{l}\text { Organisation of care } \\
\text { processes }\end{array}$ & Supporting policy & Lack of guidelines \\
\hline & Staff & Staff involvement & Poor record keeping \\
\hline & Capacities & Teamwork & Shortage of equipment, clinical supplies, \\
\hline & Resources & & and medicines \\
\hline & Structures & & Workload \\
\hline & & & Understaffing \\
\hline & & & Frequent staff rotation \\
\hline & & & Demoralised staff \\
\hline \multirow{3}{*}{$\begin{array}{l}\text { Economic and political } \\
\text { context }\end{array}$} & Financial arrangements & National policy & \multirow{3}{*}{$\begin{array}{l}\text { Deficiencies in service provision for maternity } \\
\text { care }\end{array}$} \\
\hline & Regulations & Medico-legal duty & \\
\hline & Policies & & \\
\hline
\end{tabular}

feasibility, credibility, accessibility, and attractiveness [6]. The main advantage of the partograph was that it was seen as useful for monitoring labour and continuity of practice $[14,15]$. Neither the evidence base for its effectiveness, nor its credibility, was reported as a barrier to use. Partography was, however, perceived as time-consuming [16-20]. Differences between the available versions could be confusing [21], and their completion required training.
Difficulties tended to arise when birth attendants' graphing skills were less developed than their obstetric knowledge $[22,23]$. Identifying the latent phase of labour was a particular area of confusion [16,21,24-26], and it is possible that the simplified version of the partograph may be more likely to be completed [27]. The requirement for a certain level of literacy [28], and for translation into local languages [18] were potential barriers to uptake. Finally, you 


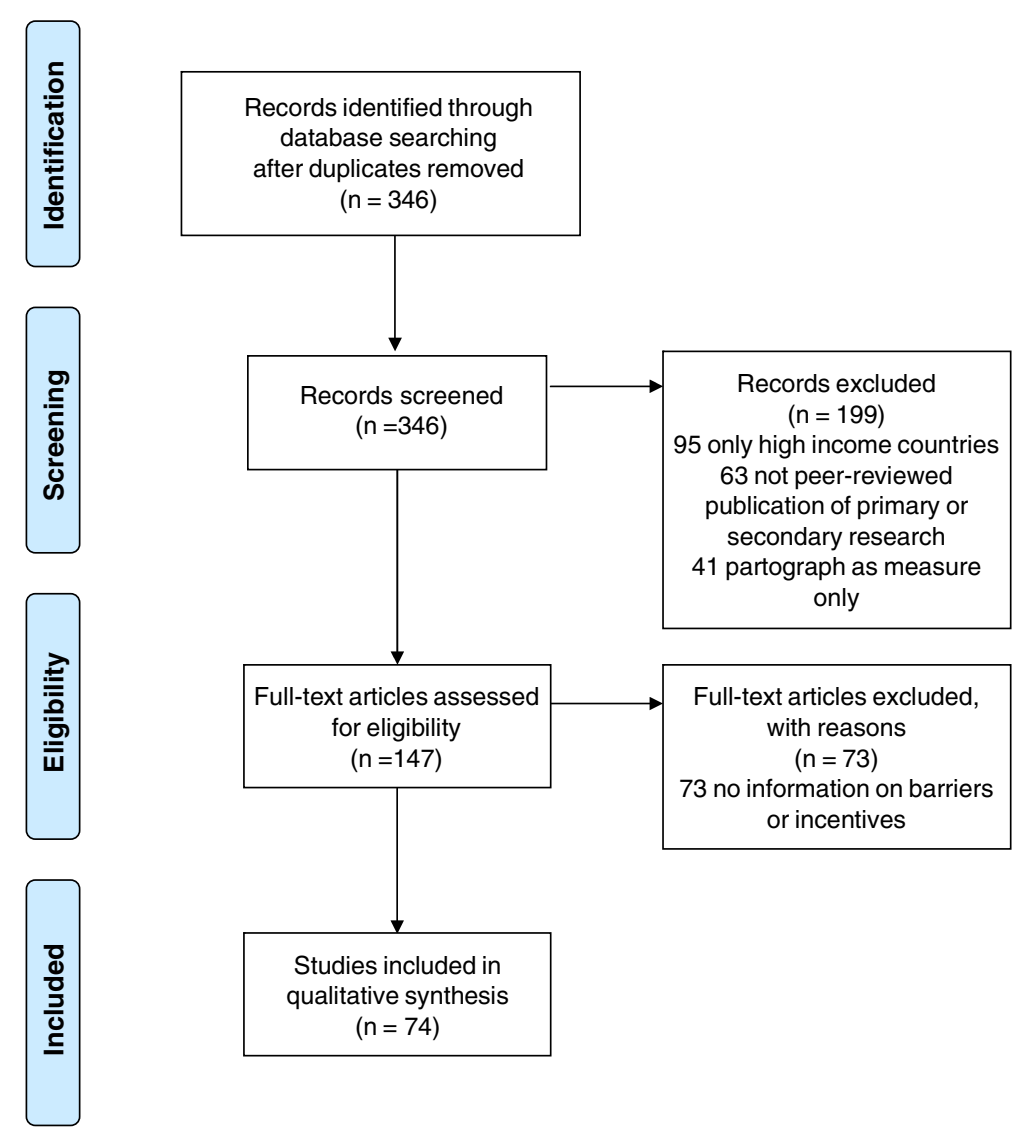

Figure 1 Flow diagram for study selection.

cannot use a partograph if you do not have it: procurement and supply chains are often problematic in LMICs, and limited availability was reported [1,15,19,29-35].

\section{Individual professional}

For individual practitioners, barriers to and incentives for change relate to their awareness, knowledge, attitude, motivation to change, and behavioural routines [6]. Effective partograph use requires health workers to "internalise its function, namely continuous monitoring, documentation and interpretation of collected information leading to early detection and prevention of neonatal and maternal complications" [16]. Where this occurred, professionals valued the partograph highly $[14,36]$. The literature included examples of instances in which this had not occurred and the partograph was not valued by staff [18], with a lack of "buy in" $[37,38]$. Low awareness of the partograph itself was an issue $[17,32,34]$, and lack of knowledge was widely reported, often within a wider deficit of knowledge and skills in maternity care $[1,15,16,18,19,32,34,35,39-46]$. Other barriers included attitude and limited confidence $[15,18]$, variation in commitment [34], and inadequate interaction with women [47].

\section{Woman}

The knowledge, skills, attitude and compliance of the woman are possible barriers to or incentives for change [6], though these were not reported relating to partograph use in LMICs, where user empowerment can be low. Training during pregnancy for women and their families to recognise prolonged labour is part of fistula prevention programmes $[48,49]$, with the potential to promote partograph use. An important factor reported as limiting partograph use was admission late in labour [26,38,47,50-52]. Clearly, some women who present later in labour may be progressing well, but others may be experiencing complications that could have been be prevented by earlier partograph monitoring.

\section{Social context}

The opinions of colleagues, organizational culture, collaboration and leadership are barriers or incentives in the social context of clinical practice [6]. Supportive culture [53], including senior leadership [16,35,54], and staff involvement $[37,55,56]$, promotes partograph use. Despite training, student midwives found partography difficult without role models and leadership from senior staff, along with facilitation in the clinical setting $[38,57]$. Senior 
midwives themselves might find the idea of learning from junior colleagues uncomfortable [44], and the social and professional barriers between midwives, physicians and managers, could also be obstacles to the process of implementation, particularly if combined with a sense of professional inadequacy [47]. Once established, supervision and monitoring $[1,15,42,58]$, and audit and evaluation $[1,16,56,59,60]$, were important to the maintenance of partograph use and quality. Without them, some social contexts allowed poor practice such as the acceptance of retrospective documentation, with completion of partographs after delivery and before discharge particularly well-described [16,35,38,47,50,57]. Contexts lacking a culture of evidence-based practice [47], or monitoring $[50,61,62]$, were also barriers to partograph use.

\section{Organisational context}

Organisation of care processes, human resources, capacities, resources and structures raises both barriers and incentives [6]. Supportive organisational policy promoted partograph use $[1,61]$, but introduction without guidelines reduced it [15]. Valuing partography as a tool for teamwork that matched provider skills with the needs of women could benefit practice [63], and involving staff in implementation was helpful [55,64]. Care processes such as the requirement for duplicate recording in notes and partographs reduce completion [1], but low use might also represent a wider deficit in record-keeping [35,59]. The most commonly reported organisational barriers were understaffing and high workload $[1,15,19,32,35,44,61,62,65]$. Frequent staff rotation [47], and job dissatisfaction also limited use [65]. Key barriers related to broader deficiencies in obstetric care [65], including shortage of equipment for measurement [28], oxytocin for labour augmentation [17], and other clinical equipment and supplies [44,50,53].

\section{Economic and political context}

Clinical care is a reflection of the broader economic and political environment. Barriers or incentives include financial arrangements, regulations and policies [6]. Supportive national policy assisted partograph use [62], and its requirement as a medico-legal duty could emphasise its value [61]. The same duty of completion could, however, lead to recording of false data and a missed opportunity to improve clinical care [38]. Studies referred to the wider deficit in comprehensive systems for obstetric care and the WHO study noted underlying "geographical, economic, political and sociocultural" constraints [66].

\section{Discussion}

\section{Main findings}

Partography is well established in many settings, and the synthesis aimed to help us consolidate its use. Many reported barriers to and incentives for partograph use affected local practice in LMICs. These related to the professional and practice environment of obstetric care, rather than to the evidence base. Partographs needed to be available, with appropriate equipment and clinical supplies for assessing progress in labour, and the resources to provide recommended interventions. Professionals might lack awareness, knowledge and training, and under-value partographs, seeing completion as complex and time-consuming rather than assisting good practice. A supportive professional environment from peers and leaders, with quality assurance systems, promoted partograph use. Adaptation to the local context was often needed in terms of both language and clinical practice. Empowerment of women to expect better care, with delivery at health facilities and earlier admission, would be likely to increase future partograph use.

\section{Strengths and limitations}

Ours was a comprehensive review of partograph use in LMICs, based on reports of practice published in journals. The exclusion of non-English papers resulted in low representation of experience from francophone Africa and Latin America, and there may also have been material in the grey literature that was not examined. However, it is likely that many of the barriers and incentives would be common to health facilities in different places, and the focus on local assessment could identify relevant factors.

\section{Interpretation}

A multilevel approach to assessing barriers to and incentives for change [6] can provide a framework to improve partograph implementation and use. Local barriers and incentives from this review could be assessed by seeking information from healthcare workers, or observing clinical practice, and matched to methods for changing practice [10]. Training is most frequently reported as a tool for better practice and lack of skills and knowledge are common. While educational initiatives address this, other approaches to changing behaviour, focused on cognition, attitude and motivation, may be important for the individual professional [6]. Shaping the social network and developing leadership, with assessment and accountability for clinical quality improvement, could support the application of learning. Wider organisational opinion needs to be supportive of partography, but deficiencies in staffing, supplies and maternity care, both limit partograph use, and reduce the likelihood of evidence-based practice.

Further consideration of new technologies for the partograph may address some barriers and support use [1]. For example, electronic partographs - into which raw data are entered and internally formatted and charted could overcome graphing difficulties. Linking electronic partography with a management protocol could prompt 
clinical assessment and action, overcoming gaps in staff knowledge. The PartoPen, a digital pen partograph, is another technology being tested to assist data validation and provide clinical prompts and decision support [67]. The need for training, supervision and follow-up remains, but future use of e-learning could increase knowledge [23]. New opportunities for communication, education and decision support through mobile devices (mHealth) could also provide maternity care workers with remote advice and produce data for feedback and audit [68]. These technologies and other adaptations of the partograph, in order "to be of optimal value to the users" [14] in different settings, could facilitate use.

Partographs can only improve outcomes within an effective maternity care system. Essentials for their use are skilled birth attendants working with a labour management protocol, with appropriate training, supervision and monitoring for quality assurance, and supportive policy [1]. Deficiencies in obstetric care and health systems were recurrent themes in this review. Further evaluation of the place of the partograph in maternity care should shape strategy as services develop in low resource settings, using the body of evidence on partograph use in LMICs to inform appropriate and optimal use in different settings with varying resources, and to identify remaining evidence gaps for future research.

\section{Conclusion}

Partographs are often underused in low- and middleincome countries. Reported barriers to and incentives for use have been reviewed as a basis for local assessment, relating to the innovation, woman, individual professional, and social, organisational and economic and political contexts. Identifying local barriers and incentives should inform strategies to improve use. Emerging technologies to support electronic partograph recording, with clinical prompts and remote decision support, may also address some barriers. The thresholds for essential maternity care at which the partograph adds value should be examined as services develop in low resource settings.

\section{Abbreviations \\ LMICs: Low and middle income countries, as defined by the World Bank 2012; WHO: World Health Organization}

\section{Competing interests}

The authors declare that they have no competing interests.

\section{Authors' contributions}

EO carried out the systematic review and drafted the manuscript. DO helped in the conception and design, supervised the review, and critically revised the manuscript. Both authors read and approved the final manuscript.

\section{Authors' information}

EO is a consultant in public health. DO is a paediatrician and public health researcher, Wellcome Trust Senior Research Fellow in Clinical Science, with a particular interest in women's and children's health in low and middle income countries.

\section{Acknowledgements}

We thank Anthony Costello and colleagues at the UCL Institute for Global Health, and colleagues at the Society for Nutrition, Education and Health Action (SNEHA) in Mumbai, for discussions on the thinking behind the paper.

\section{Funding}

D Osrin is supported by The Wellcome Trust (091561/Z/10/Z).

Received: 17 April 2014 Accepted: 11 August 2014

Published: 16 August 2014

\section{References}

1. Mathai M: The partograph for the prevention of obstructed labor. Clin Obstet Gynecol 2009, 52(2):256-269.

2. Bulatao RA, Ross JA: Rating maternal and neonatal health services in developing countries. Bull World Health Organ 2002, 80:721-727.

3. World Health Organization: World Health Organization partograph in management of labour. Lancet 1994, 343(8910):1399-1404.

4. Lavender T, Hart A, Smyth RMD: Effect of partogram use on outcomes for women in spontaneous labour at term. Cochrane Database Syst Rev 2013, 7. CD005461.

5. Higgins M, Farine D: Assessment of labor progress. Expert Rev Obstet Gynecol 2013, 8(1):83-95.

6. Grol R, Wensing M: What drives change? Barriers to and incentives for evidence-based practice. Med J Aust 2004, 180:557-S60.

7. Schuiling KD: The need for research on the use of the partogram and its effect on maternal infant outcomes. Int J Childbirth 2012, 2(1):3-4.

8. Grol R, Bosch M, Hulscher M, Eccles M, Wensing M: Planning and studying improvement in patient care: the use of theoretical perspectives. Milbank Q 2007, 85(1):93-138.

9. Grol R, Grimshaw J: From best evidence to best practice: effective implementation of change in patients' care. Lancet 2003, 362:1225-1230.

10. National Institute for Health and Clinical Excellence: How to change practice. Understand, identify and overcome barriers to clinical change. 2007:N1429. ISBN 1-84929-565-3.

11. National Institute of Clinical Studies: Identifying barriers to evidence uptake. 2006. AcBN 60095459804

12. Philpott RH: Graphic records in labour. Br Med J (Clin Res Ed) 1972, 4:163-165.

13. Moher D, Liberati A, Tetzlaff J, Altman DG, The PRISMA Group: Preferred Reporting Items for Systematic Reviews and Meta-Analyses: The PRISMA Statement. PLoS Med 2009, 6(6):e1000097.

14. Pettersson KOP, Svensson ML, Christensson K: The lived experiences of autonomous Angolan midwives working in midwifery-led, maternity units. Midwifery 2001, 17(2):102-114.

15. Ogwang S, Karyabakabo Z, Rutebemberwa E: Assessment of partogram use during labour in Rujumbura Health Sub District, Rukungiri District, Uganda. Afr Health Sci 2009, 9(Suppl 1):S27-S34.

16. Asp G, Sandberg J, Ezechi O, Odberg Pettersson K: Challenges of immediate newborn care in maternity units in Lagos, Nigeria: An observational study. J Obstet Gynaecol 2011, 31(7):612-616.

17. Fatusi $A O$, Makinde ON, Adeyemi AB, Orji EO, Onwudiegwu U: Evaluation of health workers' training in use of the partogram. Int I Gynaecol Obstet 2008, 100(1):41-44

18. Gao Y, Barclay L: Availability and quality of emergency obstetric care in Shanxi Province, China. Int J Gynaecol Obstet 2010, 110(2):181-185.

19. Opiah MM, Ofi $A B$, Essien EJ, Monjok E: Knowledge and utilization of the partograph among midwives in the Niger Delta Region of Nigeria. Afr J Reprod Health 2012, 16(1):125-132.

20. Yisma E, Dessalegn B, Astatkie A, Fesseha N: Knowledge and utilization of partograph among obstetric care givers in public health institutions of Addis Ababa, Ethiopia. BMC Pregnancy Childbirth 2013, 13:17.

21. van Bogaert LJ: The partogram. S Afr Med J 2003, 93(11):830-833.

22. Harvey S, Ayabaca P, Bucagu M, Djibrina S, Edson W, Gbangbade S, McCaw-Binns A, Burkhalter B: Skilled birth attendant competence: an initial assessment in four countries, and implications for the Safe Motherhood movement. Int J Gynaecol Obstet 2004, 87:203-210.

23. Lavender T, Omoni G, Lee K, Wakasiaki S, Campbell M, Watiti J, Mathai M: A pilot quasi-experimental study to determine the feasibility of implementing a partograph e-learning tool for student midwife training in Nairobi. Midwifery 2013, 29(8):876-884 
24. Hofmeyr GJ: Evidence-based intrapartum care. Best Pract Res Clin Obstet Gynaecol 2005, 19(1):103-115.

25. Hofmeyr GJ, Haws RA, Bergstrom S, Lee ACC, Okong P, Darmstadt GL, Mullany LC, Oo EKS, Lawn JE: Obstetric care in low-resource settings: what, who, and how to overcome challenges to scale up? Int I Gynaecol Obstet 2009, 107(Suppl 1):S21-S45.

26. Pettersson KO, Svensson ML, Christensson K: Evaluation of an adapted model of the World Health Organization partograph used by Angolan midwives in a peripheral delivery unit. Midwifery 2000, 16(2):82-88.

27. Mathews JE, Rajaratnam A, George A, Mathai M: Comparison of two World Health Organization partographs. Int J Gynaecol Obstet 2007 96(2):147-150

28. Dietsch E, Mulimbalimba-Masururu L: The Experience of Being a Traditional Midwife: Living and Working in Relationship With Women. J Midwifery Womens Health 2011, 56(2):161-166.

29. Fawole AO, Adekanle DA, Hunyinbo Kl: Utilization of the partograph in primary health care facilities in southwestern Nigeria. Niger J Clin Pract 2010, 13(2):200-204.

30. Fikree FF, Mir AM, Haq I-u: She may reach a facility but will still die! An analysis of quality of public sector maternal health services, District Multan, Pakistan. J Pak Med Assoc 2006, 56(4):156-163.

31. Maimbolwa MC, Ransjo-Arvidson AB, Ng'andu N, Sikazwe N, Diwan VK: Routine care of women experiencing normal deliveries in Zambian maternity wards: a pilot study. Midwifery 1997, 13(3):125-131.

32. Oladapo OT, Daniel OJ, Olatunji AO: Knowledge and use of the partograph among healthcare personnel at the peripheral maternity centres in Nigeria. J Obstet Gynaecol 2006, 26(6):538-541.

33. Sharma MP, Soni SC, Bhattacharya M, Datta U, Gupta S, Nandan D: An assessment of institutional deliveries under JSY at different levels of health care in Jaipur District, Rajasthan. Indian J Public Health 2009, 53(3):177-182

34. Umezulike AC, Onah HE, Okaro JM: Use of the partograph among medical personnel in Enugu, Nigeria. Int J Gynaecol Obstet 1999, 65(2):203-205.

35. Begum F, Khan MAT: The partograph in practice. Bangladesh Journal of Obstetrics and Gynecology 1998, 13(1):21-27.

36. Badjie B, Kao CH, Gua M, Lin KC: Partograph use among midwives in the Gambia. Afr J Midwifery Womens Health 2013, 7(2):65-69.

37. Gans-Lartey F, O'Brien BA, Gyekye FO, Schopflocher D: The relationship between the use of the partograph and birth outcomes at Korle-Bu teaching hospital. Midwifery 2013, 29(5):461-467.

38. Lavender T, Omoni G, Lee K, Wakasiaka S, Waitit J, Mathai M: Students' experiences of using the partograph in Kenyan labour wards. Afr J Midwifery Womens Health 2011, 5(3):117-122

39. Dohbit J, Nana N, Foumane P, Mboudou E, Mbu RE, Leke R: A survey of the knowledge, attitude and practice of the labour partogramme among health personnel in seven peripheral hospitals in Yaounde, Cameroon. Clinics in Mother and Child Health 2010, 7(1):1215-1219.

40. Dwivedi S, Singh R, Piang LK, Dhar N, Adhish V, Nandan D: Indigenous system of medicine lady doctors and general nurse midwives in operationalization of $24 \times 7$ services under NRHM in selected districts of Uttar Pradesh. Indian J Public Health 2009, 53(3):161-165.

41. Fawole AO, Hunyinbo Kl, Adekanle DA: Knowledge and utilization of the partograph among obstetric care givers in south west Nigeria. Afr J Reprod Health 2008, 12(1):22-29.

42. Kim YM, Tappis H, Zainullah P, Ansari N, Evans C, Bartlett L, Zaka N, Zeck W: Quality of caesarean delivery services and documentation in first-line referral facilities in Afghanistan: a chart review. BMC Pregnancy Childbirth 2012, 12:14.

43. Osungbade KO, Oginni SA, Olumide EAA, Owoaje ET: Clinical audit of intra-partum care at secondary health facilities in Nigeria. Niger J Clin Pract 2010, 13(2):210-214

44. Qureshi Z, Sekadde- Kigondu C, Mutiso S: Rapid assessment of partograph utilisation in selected maternity units in Kenya. East Afr Med J 2010, 87(6):235-241.

45. Yisma E, Dessalegn B, Astatkie A, Fesseha N: Completion of the modified World Health Organization (WHO) partograph during labour in public health institutions of Addis Ababa, Ethiopia. Reprod Health 2013, 10:23.

46. McDermott J, Beck D, Buffington ST, Annas J, Supratikto G, Prenggono D, Sri Ekonomi FM, Achadi E: Two models of in-service training to improve midwifery skills: how well do they work? J Midwifery Womens Health 2001, 46(4):217-225
47. Christensson K, Pettersson KO, Bugalho A, Cunha, Manuela M, Dgedge C, Johansson $E$, Bergstrom S: The challenge of improving perinatal care in settings with limited resources. Observations of midwifery practices in Mozambique. Afr J Reprod Health 2006, 10(1):47-6.

48. de Bernis L: Obstetric fistula: Guiding principles for clinical management and programme development, a new WHO guideline. Int J Gynaecol Obstet 2007, 99(0):S117-S121

49. MacDonald P, Stanton ME: USAID program for the prevention and treatment of vaginal fistula. Int J Gynaecol Obstet 2007, 99(Supplement 1):S112-S116.

50. Delvaux T, Ake-Tano O, Gohou-Kouassi V, Bosso P, Collin S, Ronsmans C: Quality of normal delivery care in Cote d'Ivoire. Afr J Reprod Health 2007 11(1):22-32.

51. Kambarami RA, Chirenje M, Rusakaniko S: Situation analysis of obstetric care services in a rural district in Zimbabwe. Cent Afr J Med 2000, 46(6):154-157

52. Matthews MK, Walley RL: Working with midwives to improve maternal health in rural Ghana. CJMRP 2004, 3(3):24-33.

53. Duysburgh E, Zhang WH, Ye M, Williams A, Massawe S, Sie A, Williams J, Mpembeni R, Loukanova S, Temmerman M: Quality of antenatal and childbirth care in selected rural health facilities in Burkina Faso, Ghana and Tanzania: similar finding. Trop Med Int Health 2013, 18(5):534-547.

54. Theron GB: Effect of the maternal care manual of the perinatal education programme on the ability of midwives to interpret antenatal cards and partograms. J Perinatol 1999, 19 (6 Pt 1):432-435.

55. Kongnyuy EJ, Mlava G, van den Broek N: Establishing standards for obstructed labour in a low-income country. Rural Remote Health 2008, 8(3):1022.

56. Mercer SW, Sevar K, Sadutshan TD: Using clinical audit to improve the quality of obstetric care at the Tibetan Delek Hospital in North India: a longitudinal study. Reprod Health 2006, 3:4.

57. Mathibe-Neke JM: Facilitation of midwifery students regarding utilisation of a partograph. Afr J Nurs Midwifery 2009, 11(1):34-47.

58. Tsu VD, Coffey PS: New and underutilised technologies to reduce maternal mortality and morbidity: What progress have we made since Bellagio 2003? BJOG 2009, 116(2):247-256.

59. Fesseha N, Getachew A, Hiluf M, Gebrehiwot Y, Bailey P: A national review of cesarean delivery in Ethiopia. Int J Gynaecol Obstet 2011, 115(1):106-111.

60. Strand RT, de Campos PA, Paulsson G, de Oliveira J, Bergstrom S: Audit of referral of obstetric emergencies in Angola: a tool for assessing quality of care. Afr J Reprod Health 2009, 13(2):75-85.

61. Buchmann EJ, Pattinson RC, Nyathikazi N: Intrapartum-related birth asphyxia in South Africa-lessons from the first national perinatal care survey. S Afr Med J 2002, 92(11):897-901.

62. Nyamtema AS, Urassa DP, Massawe S, Massawe A, Lindmark G, van Roosmalen J: Partogram use in the Dar es Salaam perinatal care study. Int J Gynaecol Obstet 2008, 100(1):37-40.

63. Orhue A, Aziken ME, Osemwenkha AP: Partograph as a tool for team work management of spontaneous labor. Niger J Clin Pract 2012, 15(1):1-8

64. Floyd L: Helping midwives in Ghana to reduce maternal mortality. Afr J Midwifery Womens Health 2013, 7(1):34-38.

65. Nyango DD, Mutihir JT, Laabes EP, Kigbu JH, Buba M: Skilled attendance: the key challenges to progress in achieving MDG-5 in north central Nigeria. Afr J Reprod Health 2010, 14(2):129-138.

66. World Health Organization: The application of the WHO partograph in the management of labour. Report of a WHO multicentre study 1990-91. Geneva: World Health Organization; 1994.

67. Underwood H: The PartoPen: Maternal Health. Developing Countries. Digital Pens. [http://www.partopen.com/]

68. Speciale AM, Freytsis M: mHealth for Midwives: A Call to Action. J Midwifery Womens Health 2013, 58(1):76-82.

doi:10.1186/1471-2393-14-281

Cite this article as: Ollerhead and Osrin: Barriers to and incentives for achieving partograph use in obstetric practice in low- and middleincome countries: a systematic review. BMC Pregnancy and Childbirth $201414: 281$ 